\title{
功能化石墨烯负载毛葶乙素抗肿瘤制剂的研究
}

\author{
徐志远 ${ }^{a, b, c}$ 李永军 $b \quad$ 史 萍 $*, a$ 王博婵 ${ }^{a}$ 黄晓宇 $*, b$ \\ ( ${ }^{a}$ 华东理工大学生物反应器工程国家重点实验室 上海 200237) \\ ( ${ }^{b}$ 中国科学院上海有机化学研究所 上海 200032) \\ (c 华东理工大学材料科学与工程学院 上海 200237)
}

\begin{abstract}
摘要 首先通过改进的 Hummers 法制备了氧化石墨烯(GO), 然后通过酰胺化反应将端基为氨基的六臂聚乙二醇(PEG) 连接到氧化石墨烯的表面, 以改善其水溶性和生物相容性. 原子力显微镜(AFM)数据表明所制备的 GO-PEG 的尺寸小 于 $250 \mathrm{~nm}$, 稳定性试验证明 GO-PEG 在水和 PBS 缓冲液中可以很好地分散. 利用制备的 GO-PEG 作为药物载体, 通过 物理共混的方法负载了疏水性抗肿瘤药物——毛楞乙素. 紫外光谱法测得载药率为 $18.8 \%$. 选择肺癌细胞 A549 和乳腺 癌细胞 MCF-7 对载药体系的细胞毒性进行了研究, 结果表明即使在高达 $100 \mathrm{mg} / \mathrm{L}$ 的浓度下培养 $48 \mathrm{~h}, \mathrm{GO}-\mathrm{PEG}$ 载体对 两种细胞仍然具有很小的毒性(相对细胞存活率 $>85 \%$ ), 而通过载体负载毛颌乙素后的疗效有所增强, 对癌细胞具有 更大的杀伤作用.
\end{abstract}

关键词 氧化石墨烯; 毛荳乙素; 药物载体; 细胞存活率

\section{Functionalized Graphene Oxide as a Nanocarrier for Loading and Delivering of Eriocalyxin B}

\author{
Xu, Zhiyuan ${ }^{a, b, c}$ \\ Li, Yongjun ${ }^{b}$ \\ Shi, Ping ${ }^{*, a}$ \\ Wang, Bochan ${ }^{a}$ \\ Huang, Xiaoyu ${ }^{*, b}$ \\ ( ${ }^{a}$ State Key Laboratory of Bioreactor Engineering, East China University of Science and Technology, Shanghai 200237) \\ $\left({ }^{b}\right.$ Shanghai Institute of Organic Chemistry, Chinese Academy of Sciences, Shanghai 200032) \\ ( ${ }^{c}$ School of Materials Science and Engineering, East China University of Science and Technology, Shanghai 200237)
}

\begin{abstract}
Graphene oxide (GO) was firstly prepared by modified Hummers method. In order to improve its water solubility and biocompatibility, 6-arm PEG was grafted to GO via a facile amidation reaction. The size of obtained GO-PEG was less than $250 \mathrm{~nm}$. Stability test indicated the good dispersibility of GO-PEG in water and PBS buffer. Furthermore, eriocalyxin B, a widely used cancer chemotherapy drug, is adsorbed onto GO-PEG via physical blending with a drug loading ratio of $18.8 \%$ obtained by UV spectrum. Lung cancer cell A549 and breast cancer cell MCF-7 were selected to study the cytotoxicity of GO-PEG/eriocalyxin B, GO-PEG, and free eriocalyxin B. The results demonstrated that GO-PEG nano-carrier possessed low toxicity (relative cell viability $>85 \%$ ), even cultivated for $48 \mathrm{~h}$ at a relatively high concentration of $100 \mathrm{mg} / \mathrm{L}$. Compared to pure drug, GO-PEG/eriocalyxin B nanocarrier shows higher cytotoxicity in A549 and MCF-7 cells.

Keywords graphene oxide; eriocalyxin B; drug delivery; cell viability
\end{abstract}

我国具有丰富的天然资源，一些天然化合物已被证 明具有较强的抗肿瘤作用, 如毛葶乙素(eriocalyxin B). 它是从毛䓯香茶菜中提取分离得到的贝壳杉烷二萜类 化合物, 为抗肿瘤药物冬凌草甲素的类似物 ${ }^{[1]}$, 其化学 结构如图 1 所示. 研究表明, 毛若乙素对乳腺癌、结肠 癌、白血病、肺癌、膀胱癌及肝癌等多种肿瘤细胞均有 较好的增殖抑制作用, 是一种潜在的抗肿瘤活性物质 ${ }^{[2]}$.
然而由于其水溶性差、生物利用度低等原因 ${ }^{[3]}$, 其应用 一直受到限制. 因此，寻找合适的药物载体，提高毛荳 乙素的生物利用度具有重要的意义.

许多研究小组致力于改善毛萝乙素的水溶性，通过 将水溶性好的小分子化合物连接到药物分子上，期望得 到水溶性较好的具有临床应用前景的新药化合物. 中国 科学院昆明植物研究所孙汉董等 ${ }^{[1,3]}$ 合成了一系列毛䔅

*E-mail: ship@ecust.edu.cn; xyhuang@mail.sioc.ac.cn

Received June 7, 2013; revised June 18, 2013; published online June 25, 2013.

Project supported by the National Natural Science Foundation of China (Nos. 31100549, 21204098), the Shanghai Scientific and Technological Innovation Project (No. 10431903000) and the National Special Fund for State Key Laboratory of Bioreactor Engineering (No. 2060204).

国家自然科学基金(Nos. 31100549, 21204098)、上海市生物医药科技重点(No. 10431903000)和国家重点实验室专项经费(No. 2060204)资助项目. 


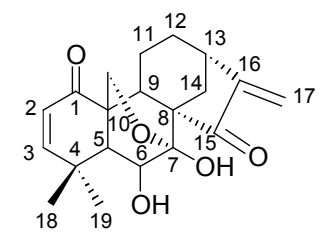

图 1 毛䒓乙素的化学结构

Figure 1 Chemical structure of eriocalyxin B

乙素的衍生物, 并研究了其对肿瘤细胞的细胞毒性. 然 而, 基于小分子化学修饰改善药物的水溶性的方法, 在 药物的研究以及应用过程中, 常会存在一些影响药物发 挥其应有的作用以及影响药物应用的因素 ${ }^{[4]}$. 如药代动 力学性质不理想而使药物的吸收受到影响, 或是由于本 身结构的特点而引起代谢速度过慢或者过快等情况; 此 外, 药物如溶解性能差、化学稳定性差、有不良的气味 或者味道以及对机体产生刺激性等.

纳米药物传输系统(nanoparticle drug delivery system, NDDS)可以有效地避免以上的缺点, 特定尺度 $(50 \sim 200$ $\mathrm{nm})$ 的纳米粒子对肿瘤组织具有增强渗透和停留效应 (EPR, enhanced permeability and retention effect), 能够 渗入到肿瘤组织的内部并聚集, 因此具有更好的效果, 可以提高化疗药物的药效, 降低其毒副作用 ${ }^{[5]}$. 基于石 墨烯为载体的载药体系是近几年发展起来的较为新颖 的纳米载药体系, 可以达到高的载药量, 更好地提高疏 水性药物的功效 ${ }^{[6]}$.

石墨烯是由碳原子以 $\mathrm{sp}^{2}$ 杂化连接形成的具有理想 二维结构的单原子层原子晶体 ${ }^{[7]}$, 在生物医学领域应用 较多的石墨烯衍生物主要是功能化的氧化石墨烯. 氧化 石墨烯(graphene oxide, GO)通常是由石墨经化学氧化、 超声制备获得, 因氧化条件不同, 所获得的氧化石墨烯 尺寸一般在几十纳米到几百纳米乃至微米之间. 氧化石 墨烯含有大量的含氧活性基团, 如羧基、羟基与环氧基 等, 因此提高了其亲水性, 同时有利于化学功能化修饰, 以达到在不同领域应用的目的 ${ }^{[8]}$. 将生物相容性良好的 聚合物分子, 如聚乙二醇( $\mathrm{PEG}$ )、壳聚糖(CS)等接枝到氧 化石墨烯表面有利于改善其生物相容性以达到生物领 域的应用 ${ }^{[9]}$.

本工作中, 以 $\mathrm{PEG}$ 功能化的氧化石墨烯为载体, 对 毛蔩乙素进行了负载试验, 并研究了所得到的纳米药物 载体的细胞毒性, 旨在发展载药量高的生物相容性药物 载体, 提高抗肿瘤药物的生物利用度, 并提高疗效.

\section{1 结果与讨论}

\subsection{PEG 功能化 GO 的制备及表征}

载体 GO-PEG 的合成及药物的负载路线如 Scheme
1 所示. 首先将六臂 PEG 端基的羟基转化为氨基，以提 高其与 $\mathrm{GO}$ 上羧基的反应效率. 其具体过程为先将 PEG 上的羟基转变为叠氮基团, 再将叠氮转变为氨基 ${ }^{[10]}$.

采用改性的 Hummers 法制备氧化石墨, 两步法可 以提高氧化程度. 红外光谱图(图 2)中, $3400 \mathrm{~cm}^{-1}$ 处羟 基的大吸收峰、 $1732 \mathrm{~cm}^{-1}(\mathrm{C}=\mathrm{O})$ 及 $1622 \mathrm{~cm}^{-1}(\mathrm{C}=\mathrm{C})$ 处的特征峰的出现证实了石墨的氧化.

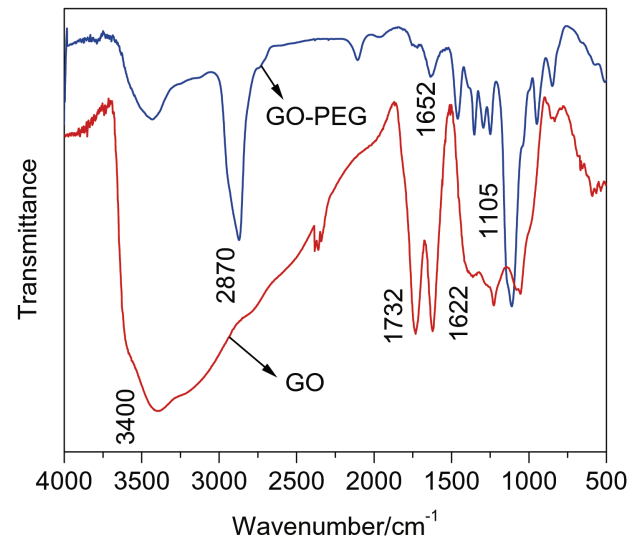

图 2 石墨与氧化石墨烯的红外光谱图

Figure 2 FT-IR spectra of graphite and graphene oxide

$\mathrm{GO}$ 与 $\mathrm{PEG}$ 反应前, 首先在 $\mathrm{NaOH}$ 溶液中将 $\mathrm{GO}$ 上 的羟基转化为羧基, 以提高与 $\mathrm{PEG}$ 上氨基的反应效率. 由于强碱的存在, $\mathrm{GO}$ 出现沉淀, 反应过程中 $\mathrm{GO}$ 的部分 还原使溶液颜色变黑. 透析除去 $\mathrm{NaOH}$ 后, $\mathrm{GO}$ 仍可以很 好地分散在水中. GO-PEG 的红外谱图中 $2870\left(\mathrm{CH}_{2}\right)$, $1652(\mathrm{NH}-\mathrm{CO})$ 和 $1105 \mathrm{~cm}^{-1}(\mathrm{C}-\mathrm{O})$ 吸收峰的出现证明 PEG 成功接枝到氧化石墨烯上. 利用 AFM 对 GO 与 GO-PEG 的形貌进行表征, 结果如图 3 所示. GO 的表面 较为光滑平整，尺寸基本处于 $50 \sim 450 \mathrm{~nm}$ 之间，厚度为 $0.8 \sim 1.4 \mathrm{~nm}$, 由于 $\mathrm{PEG}$ 的接入以及接枝过程中的超声 处理, GO-PEG 的表面较为粗糙且具有褶狓, 尺寸减小 为 $50 \sim 300 \mathrm{~nm}$, 厚度增加为 $6.0 \sim 8.5 \mathrm{~nm}$.

将浓度为 $0.5 \mathrm{mg} / \mathrm{mL}$ 的 GO 与 GO-PEG 分别分散在 去离子水与 PBS 缓冲液中, 如图 4 所示. GO 与 GO-PEG 均可以在水中很好地分散, 静置一个月后无沉淀及聚集 现象; GO 在 PBS 缓冲液中静置 $1 \mathrm{~d}$ 后出现聚集沉淀, GO-PEG 仍可以在 PBS 缓冲液中稳定分散, 静置一个月 无聚集沉淀现象，说明向 GO 上引入水溶性好的 PEG 后, 的确可以改善其亲水性以及在盐溶液中的稳定性.

\subsection{PEG 功能化 GO 对毛䓯乙素的负载}

为了测试 PEG 功能化 GO 对毛萝乙素包封结果, 我 们先测定了毛颌乙素在乙醇中的标准曲线. 配置不同浓 度 $(1,2.5,5,10,20,40 \mathrm{mg} / \mathrm{L})$ 的毛荳乙素乙醇溶液, 测定 

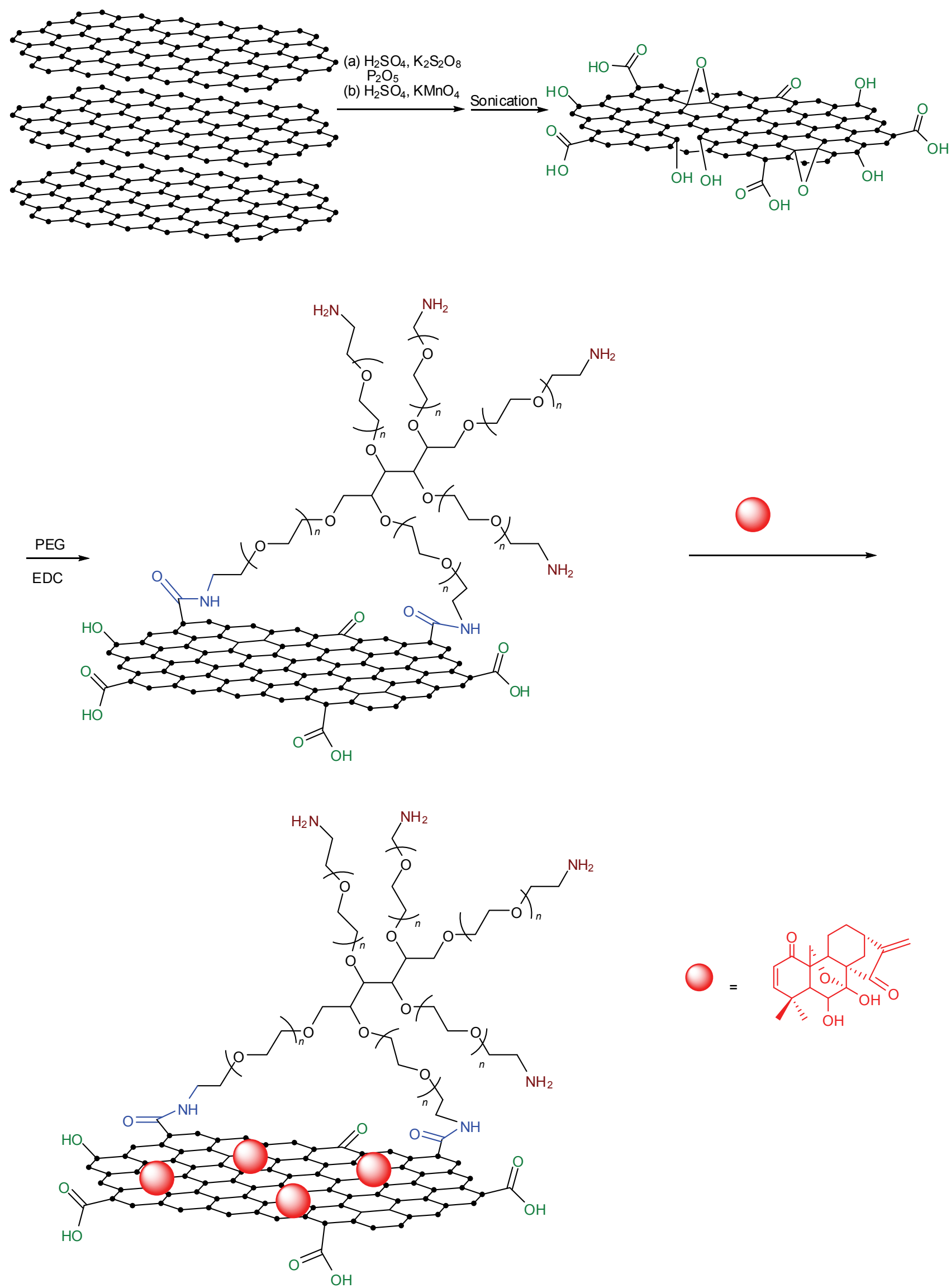

Scheme 1 

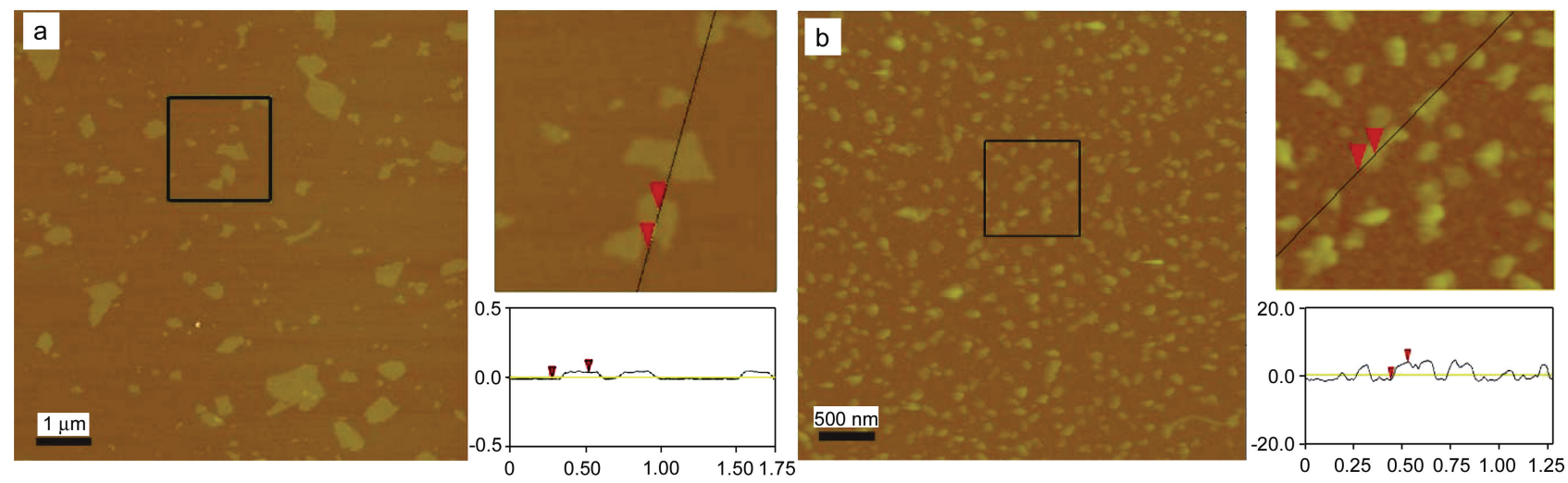

图 $3 \mathrm{GO}(\mathrm{a})$ 与 $\mathrm{GO}-\mathrm{PEG}(\mathrm{b})$ 的 $\mathrm{AFM}$ 图

Figure 3 AFM images of GO (a) and GO-PEG (b)

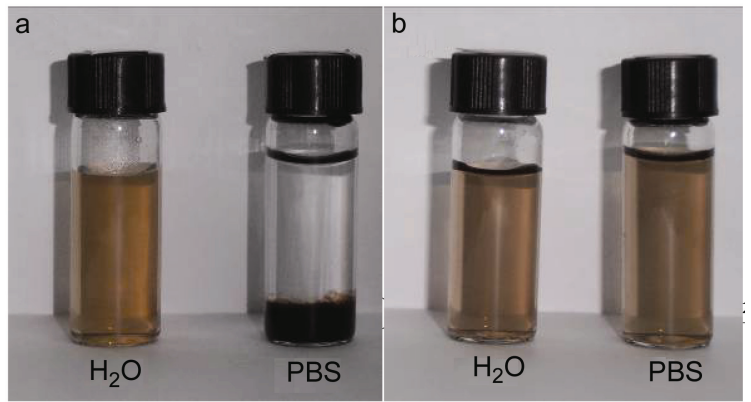

图 $4 \mathrm{GO}$ (a) 与 $\mathrm{GO}-\mathrm{PEG}$ (b) 在水与 $\mathrm{PBS}$ 中的稳定性

Figure 4 Stability of GO (a) and GO-PEG (b) in water and PBS

其在 $230 \mathrm{~nm}$ 处的吸光度, 并作浓度与吸光度的标准曲 线(图 5), 按回归法处理得标准曲线方程(Eq. 1).

$$
\text { Abs }=0.07139+0.04845 \text { [eriocalyxin B] }
$$

利用 GO-PEG 对进行毛萝乙素负载, 首先将毛萼乙 素溶于乙醇中, 与 GO-PEG 的水溶液搅拌过夜; 然后通 过离心、过滤除去未被载体吸附的毛萝乙素, 直至紫外 光谱检测不到过滤的水中毛萼乙素的吸收峰. 通过紫外 光谱中载药体系在 $230 \mathrm{~nm}$ 处的吸光度减去该波长处 GO-PEG 的吸光度, 可以推算出负载的毛颌乙素的吸光 度, 对照标准曲线即可得到毛萝乙素的浓度. 由载体所 负载的药物质量除以载体的质量, 即可算出体系的载药 率为 $18.8 \mathrm{wt} \%$.

\section{3 细胞毒性试验}

选择肺癌细胞 A549 和乳腺癌细胞 MCF-7 对载药体 系的细胞毒性进行了研究, 采用细胞增殖-毒性检测试 剂盒(CCK-8)测其细胞存活率 ${ }^{[10]}$, 结果如图 6 所示.

对于 $\mathrm{A} 549$ 细胞, 以毛葶乙素浓度为 $8 \mu \mathrm{mol} \cdot \mathrm{L}^{-1}$ 为 例，在只有毛荳乙素存在的条件下, $24 \mathrm{~h}$ 时 A549 细胞的 相对存活率为 $56.5 \%$ ，而通过 GO-PEG 负载毛墓乙素后， A549 细胞的相对存活率大幅下降为 $52.6 \%$; 培养 $48 \mathrm{~h}$ 后，前者的相对细胞存活率降低为 $28.5 \%$, 而后者降低
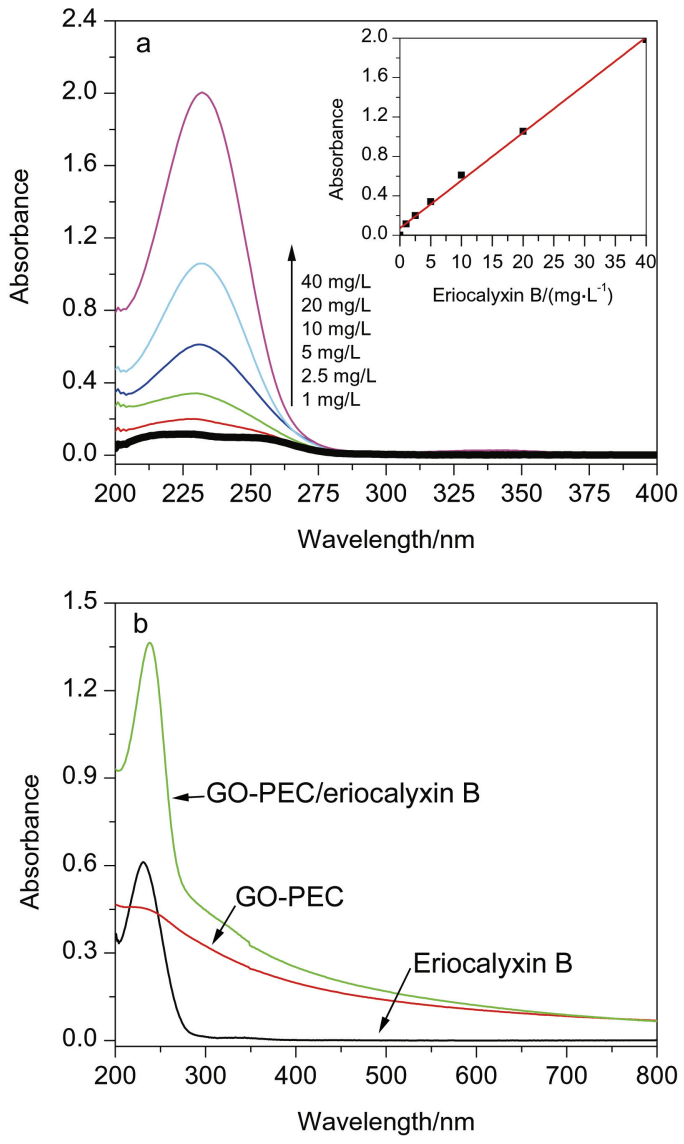

图 5 不同浓度毛莒乙素素在 $230 \mathrm{~nm}$ 处的吸光度及吸光度浓度标准曲线(a)与 GO-PEG 载药前后紫外光谱(b)

Figure 5 (a) Absorbance of eriocalyxin $\mathrm{B}$ with different concentrations and its standard curve at $230 \mathrm{~nm}$ and (b) UV spectra of GO-PEG before and after loading drug

为 $21.8 \%$. 对于 MCF-7 细胞, 以毛荳乙素浓度为 8 $\mu \mathrm{mol} \cdot \mathrm{L}^{-1}$ 为例, 在只有毛萼乙素存在的条件下, $24 \mathrm{~h}$ 时 MCF-7 细胞的相对存活率为 $73.2 \%$, 而通过 GO-PEG 负 载毛萝乙素后, MCF-7 细胞的相对存活率下降为 $68.6 \%$; 

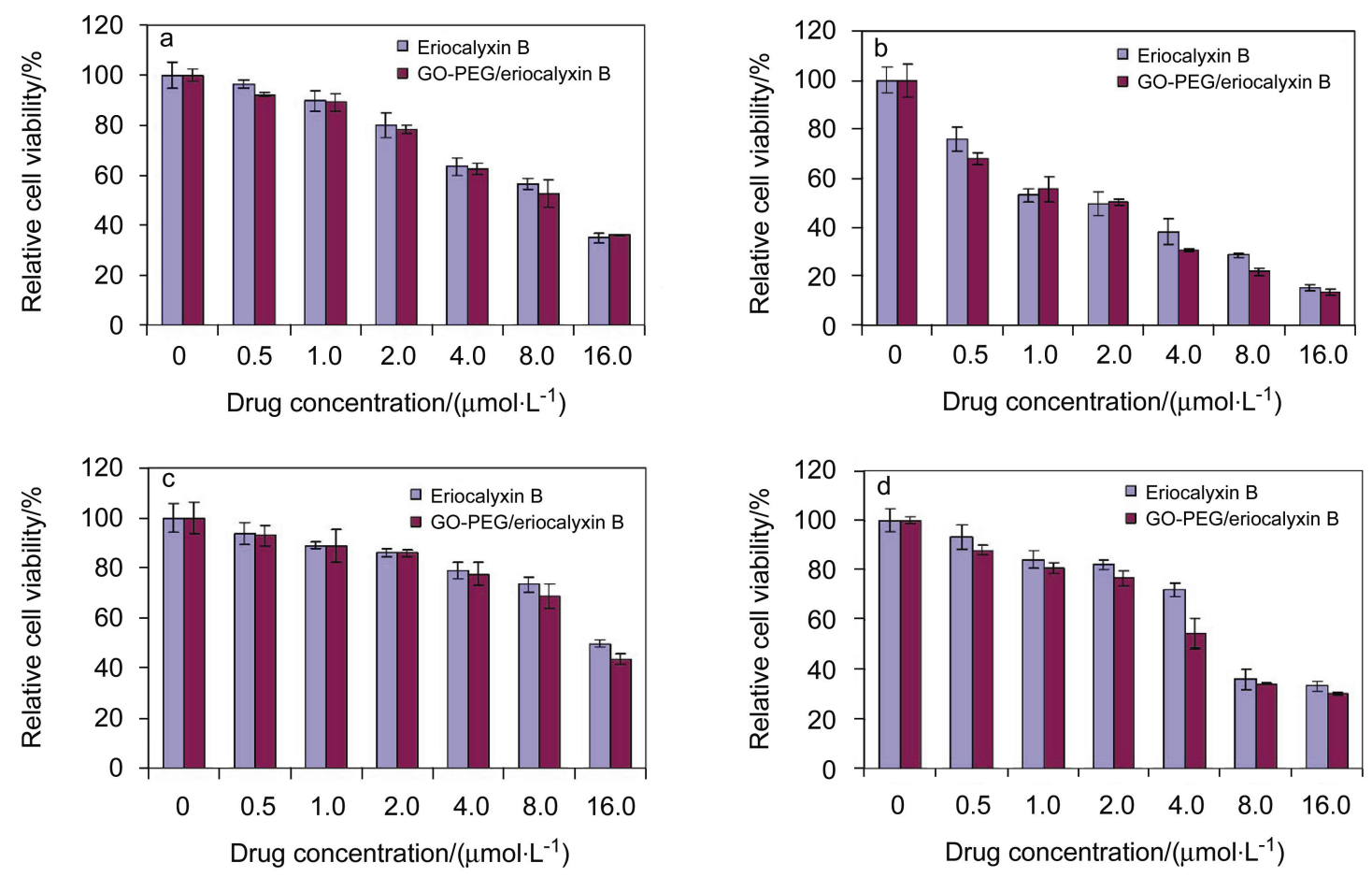

图 6 相对细胞存活率与药物浓度的关系图

Figure 6 Relative cell viability of cells at various drug concentrations (a), (b) A549 cells treated with eriocalyxin B and GO-PEG/eriocalyxin B after 24 and $48 \mathrm{~h}$, respectively; (c), (d) MCF-7 cells treated with eriocalyxin B and GO-PEG/eriocalyxin B after 24 and $48 \mathrm{~h}$, respectively
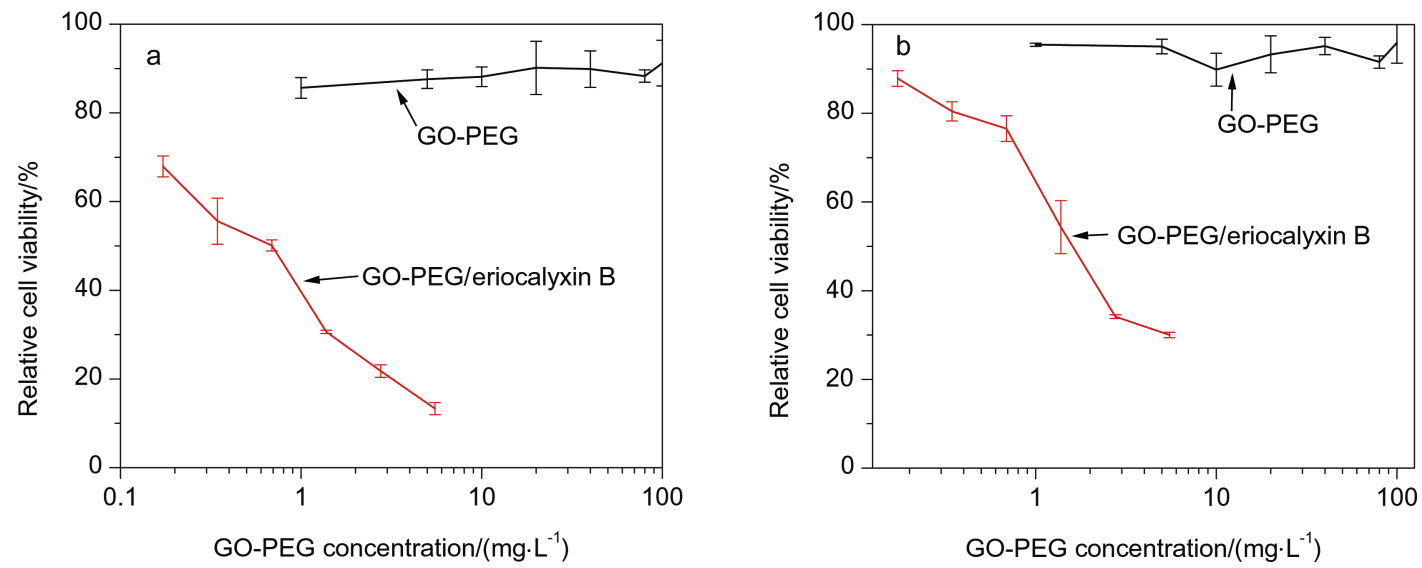

图 7 GO-PEG 载药前后(a) A549 细胞和(b) MCF-7 细胞存活率

Figure 7 Relative cell viability of A549 (a) and MCF-7 (b) before and after loading eriocalyxin B

培养 $48 \mathrm{~h}$ 后, 前者的相对细胞存活率降低为 $36 \%$, 后者 降低为 $34.2 \%$. 结果表明, 单独的毛萼乙素与 GO-PEG/ eriocalyxin B 对细胞的毒性均具有一定的时间与浓度依 赖性: 即在一定的时间与浓度范围内, 随着时间与浓度 的增加, 两者对细胞的杀伤作用越大. 与单独的毛萼乙 素相比, GO-PEG/eriocalyxin B 可以更有效地杀伤 A549 细胞和 MCF-7 细胞. 对照试验结果(图 7)表明, 即使在 GO-PEG 浓度高达 $100 \mathrm{mg} / \mathrm{L}$ 时, 培养 $48 \mathrm{~h}$ 后两种细胞 的相对存活率仍在 $85 \%$ 以上. 说明单独的 GO-PEG 药物
载体对两种细胞基本没有毒性，体系对细胞的杀伤作用 完全来自于 GO-PEG 载体所吸附的毛莒乙素.

\section{2 结论}

成功制备了药物载体 GO-PEG, 经 PEG 功能化后, 该载体具有很好的亲水性与生物相容性，在水及 $\mathrm{PBS}$ 中 均可长期稳定存在. 通过疏水相互作用与 $\pi-\pi$ 堆积作用 负载毛䒓乙素，载药率高达 $18.8 \%$. 体外实验证明，该 载药体系对 A549 细胞与 MCF-7 细胞具有很好的杀伤作 
用, 其细胞毒性明显高于单独药物. 该载药体系可以有 效地改善疏水性药物的水溶性, 提高抗肿瘤药物的生物 利用度, 具有很好的应用前景.

\section{3 实验部分}

\section{1 仪器与试剂}

红外光谱(FTIR): Nicolet AVATAR-360, 液体样品 直接用液膜法测试, 固体样品用溴化钾压片法, 所有红 外谱图累加 32 次, 分辨率为 $4.0 \mathrm{~cm}^{-1}$. 紫外分光光度计: Cary 100, $25{ }^{\circ} \mathrm{C}$ 下扫描毛蓦乙素的 $200 \sim 800 \mathrm{~nm}$ 的全谱, 测定 $230 \mathrm{~nm}$ 处的吸收强度. 原子力显微镜: 美国 Veeco 公司, Nanoscope IVa-Multimode, 将样品滴加到云母片 上, 吹干后采用轻敲模式测量. 相对细胞存活率用瑞士 Tecan 公司的多功能酶标仪测定其 $450 \mathrm{~nm}$ 处的吸光度 计算得到.

鳞片石墨(1000 目), 上海一帆石墨有限公司; 6 臂聚 乙二醇 $\left(M_{\mathrm{w}}=6000\right)$, 复旦大学提供; 毛蔩乙素 $(97 \%)$, 云 南西力生物技术有限公司; RPMI1640 与 DMEM 细胞培 养基, 美国 Invitrogen 公司, 并用以色列 BI 公司的 10\% 胎牛血清配成细胞培养液; 细胞增殖-毒性检测试剂盒 (CCK-8), 日本同仁化学研究所. 四氢呋喃(THF) 经钠 丝干燥, 氮气保护下, 经钠丝在二苯甲酮存在下回流除 水至呈紫色后蒸出, 现蒸现用. 其它试剂未注明的均未 经处理直接使用.

\section{$3.2 \mathrm{GO}$ 的制备}

采用两步法制备 $\mathrm{GO}$. 首先将 $\mathrm{K}_{2} \mathrm{~S}_{2} \mathrm{O}_{8}(2 \mathrm{~g})$ 与 $\mathrm{P}_{2} \mathrm{O}_{5}(2$ $\mathrm{g}$ )加入到 $12 \mathrm{~mL}$ 浓硫酸中, 升温到 $80{ }^{\circ} \mathrm{C}$, 称取 $2 \mathrm{~g}$ 鳞片 石墨加入上述混合液中, 并于 $80{ }^{\circ} \mathrm{C}$ 下摚拌反应 $6 \mathrm{~h}$; 反 应完成后, 使反应液降到室温并用 $0.5 \mathrm{~L}$ 去离子水稀释、 过滤, 用去离子水冲洗多次以除去残留的硫酸, 干燥. 然后称取上述预氧化的石墨 $1 \mathrm{~g}$ 加入三口瓶中, 并加入 $23 \mathrm{~mL}$ 浓硫酸, 冷却至 $0{ }^{\circ} \mathrm{C}$, 缓慢加入 $6 \mathrm{~g} \mathrm{KMnO}_{4}$ 并摚 拌, 使得体系温度不超过 $20{ }^{\circ} \mathrm{C}$; 将三口瓶移入油浴中 于 $35{ }^{\circ} \mathrm{C}$ 反应 $2 \mathrm{~h}$, 升温至 $90{ }^{\circ} \mathrm{C}$ 反应 $90 \mathrm{~min}$; 接着向三 口瓶中滴加 $46 \mathrm{~mL}$ 去离子水, 体系温度升至 $100{ }^{\circ} \mathrm{C}$ 并维 持 $20 \mathrm{~min}$; 继续加入 $140 \mathrm{~mL}$ 去离子水, 缓慢滴加 $20 \mathrm{~mL}$ $30 \% \mathrm{H}_{2} \mathrm{O}_{2}$, 体系变为亮黄色并有气泡产生. 用 $250 \mathrm{~mL}$ $1: 10 \mathrm{HCl}$ 溶液处理后过滤, 并用去离子水反复冲洗. 过滤产物加水稀释后超声处理, 并于 $12000 \mathrm{r} / \mathrm{min}$ 下离 心, 上层悬浮液即为产物. FTIR (KBr) v: 3400, 1732, $1622 \mathrm{~cm}^{-1}$.

\subsection{PEG-NH $\mathrm{N}_{2}$ 的合成}

将 PEG $\left(15 \mathrm{~g}\right.$, 约 $\left.2.5 \times 10^{-3} \mathrm{~mol}\right)$ 、甲烷磺酰氯 $(3.1 \mathrm{~g}$, $0.026 \mathrm{~mol})$ 、四氢呋喃 $(30 \mathrm{~mL})$ 加入到三口瓶中, 搅拌溶
解. 经过 3 次抽换氮气后, 将三口瓶置于冰浴中冷却至 $0{ }^{\circ} \mathrm{C}$, 氮气保护. 用注射器滴加三乙胺 $(4.1 \mathrm{~mL}, 0.03$ $\mathrm{mol})$, 室温下, 傥拌过夜. 向反应液中加入 $32 \mathrm{~mL} \mathrm{Na}-$ $\mathrm{HCO}_{3}(1.75 \mathrm{~g}, 0.021 \mathrm{~mol})$ 溶液与 $\mathrm{NaN}_{3}(2.1 \mathrm{~g}, 0.032 \mathrm{~mol})$, 加热使四氢呋喃挥发, 并回流 $8 \mathrm{~h}$. 冷却至室温, 用二氯 甲烷萃取三次, 合并有机相, $\mathrm{Na}_{2} \mathrm{SO}_{4}$ 除水, 过滤, 旋蒸, 干燥后得 PEG- $\mathrm{N}_{3}$ (产率 78\%). FTIR (film) $v: 2104 \mathrm{~cm}^{-1}$.

将 PEG- $N_{3}\left(5 \mathrm{~g}\right.$, 约 $\left.8.13 \times 10^{-4} \mathrm{~mol}\right)$ 溶于 $40 \mathrm{~mL}$ 甲醇 中, 加入 $10 \% \mathrm{Pd} / \mathrm{C}(100 \mathrm{mg})$, 室温下充 $\mathrm{H}_{2}$ 反应 $3 \mathrm{~d}$, 过 滤除去 $\mathrm{Pd} / \mathrm{C}$, 旋蒸, 干燥后得黄色产物(产率 97\%). FTIR (film) $v: 3560 \mathrm{~cm}^{-1}$.

\subsection{GO-PEG 的制备}

首先, 根据文献[13]报道将 $\mathrm{GO}$ 上的羟基转化为羧 基. 将改性后的 $\mathrm{GO}(100 \mathrm{~mL}$, 约 $1 \mathrm{mg} / \mathrm{mL})$ 与 $\mathrm{PEG}-\mathrm{NH}_{2}$ (300 mg)超声 $5 \mathrm{~min}$, 加入 $\mathrm{EDC} \cdot \mathrm{HCl}(30 \mathrm{mg})$, 超声 40 $\mathrm{min}$, 继续加入 $\mathrm{EDC} \cdot \mathrm{HCl}(80 \mathrm{mg})$, 搅拌过夜. 将反应混 合物放入可透过分子量为 14000 的透析袋中, 透析一周, 除去未连接到 GO 上的 PEG. FTIR (KBr) v: 2870, 1625, $1105 \mathrm{~cm}^{-1}$.

\section{5 毛萼乙素的负载}

GO-PEG $(10 \mathrm{~mL}, 0.5 \mathrm{mg} / \mathrm{mL})$ 与毛荳乙素 $(10 \mathrm{~mL}$, $0.5 \mathrm{mg} / \mathrm{mL}$ ) 的乙醇溶液室温摚拌 $12 \mathrm{~h}$, 离心除去析出的 毛䔅乙素; 通过 $10 \mathrm{kDa}$ 的过滤膜, 并反复冲洗, 除去未 被 GO 吸附的毛蓄乙素及乙醇. 制备的载药体系 GO-PEG/eriocalyxin B 于 $4{ }^{\circ} \mathrm{C}$ 下储存.

\section{6 细胞培养与体外实验}

A549 与 MCF-7 细胞分别培养在盛有 $10 \%$ 胎牛血清 的 RPMI 1640 与 DMEM 培养液的培养瓶中, 将培养瓶 置于 $37{ }^{\circ} \mathrm{C}$ 含 $5 \% \mathrm{CO}_{2}$ 的细胞培养箱中. 将细胞转移到 96 孔板中, 每孔 $100 \mu \mathrm{L}$ 培养液, 细胞密度为 $5 \times 10^{4}$ 个/ 孔. 分别加入不同浓度的 GO-PEG/eriocalyxin B 与纯的 毛䓯乙素(溶于 DMSO 中, 并用 PBS 缓冲液稀释), 其中 两种细胞所加药物浓度为 $\left(0,0.5,1,2,4,8,16 \mu \mathrm{mol} \cdot \mathrm{L}^{-1}\right)$.

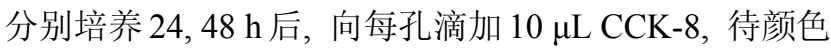
变化后在多功能酶标仪下测其吸光度, 并计算相对细胞 存活率. 对照试验为向每孔中加入不同浓度的 GO-PEG $(0,1,5,10,20,40,80,100 \mathrm{mg} / \mathrm{L})$ 培养 $48 \mathrm{~h}$, 多功能酶标 仪下测其在 $450 \mathrm{~nm}$ 处的吸光度, 并计算相对细胞存活 率.

\section{References}

[1] (a) Zhou, W. S.; Cheng, Y. X. Acta Chim. Sinica 1990, 48, 1185 (in Chinese).

(周维善, 程云行, 化学学报, 1990, 48, 1185.)

(b)Wang, J.; Hou, H. M.; Xia, Y. R.; Sun, H. D.; Pu, J. X. Chin. J. 
Pharm. 2012, 43, 194 (in Chinese).

(王健, 侯惠民, 夏怡然, 孙汉董, 普建新, 中国医药工业杂志, 2012, 43, 194.)

[2] Xia, Y. R.; Wang, J.; Zhu, J. P.; Li, D. Chin. J. Pharm. 2010, 41, 41 (in Chinese).

(夏怡然, 王健，朱金屏，陈芳，李丁，中国医药工业杂志，2010, 41, 41.)

[3] Zhao, Y.; Niu, X. M.; Qian, L. P.; Liu, Z. Y.; Zhao, Q. S.; Sun, H. D. Eur. J. Med. Chem. 2007, 42, 494

[4] (a) Ojima, I.; Geng, X. D.; Wu, X. Y., Qu, C. X.; Borella, C. P.; Xie, H. S.; Wilhelm, S. D.; Leece, B. A.; Bartle, L. M.; Goldmacher, V. S.; Chari, R. V. J. J. Med. Chem. 2002, 45, 5620.

(b) Zhao, C. W.; Zhuang, X. L.; He, P.; Xiao, C. S.; He, C. L.; Sun, J. R.; Chen, X. S.; Jing, X. B. Polymer 2009, 50, 4308.

[5] (a) Liu, Z.; Chen, K.; Davis, C.; Sherlock, S.; Cao, Q. Z.; Chen, X. Y.; Dai, H. J. Cancer Res. 2008, 68, 6625.

(b) Huang, X. W.; Guo, L. N.; Lu, G. L.; Huang, X.; Zhang, Y. Q.; Huang, X. Y. Acta Chim. Sinica 2009, 67, 1363 (in Chinese).

(黄晓炜, 顾丽娜, 陆国林, 黄啸, 张亚琴, 黄晓宇, 化学学报, 2009, 67, 1363.)

(c) Chen, S. Y.; Zhao, X. R.; Chen, J. Y.; Chen, J.; Kuznetsova, L.; Wong, S. S.; Ojima, I. Bioconjugate Chem. 2010, 21, 979.

[6] Sun, X. M.; Liu, Z.; Welsher, K.; Robinson, J. T.; Goodwin, A.; Zaric, S.; Dai, H. Nano Res. 2008, 1, 203.

[7] (a) Novoselov, K. S.; Geim, A. K.; Morozov, S. V.; Jiang, D.; Zhang, Y.; Dubonos, S. V.; Grigorieva, I. V.; Firsov, A. A. Science 2004, 306, 666 . (b) Dai, J.; Lang, M. D. Acta Chim. Sinica 2012, 70, 1237 (in Chinese).

(戴静, 郎美东, 化学学报, 2012, 70, 1237.)

(c) Deng, Y.; Li, Y. J.; Dai, J.; Lang, M. D.; Huang, X. Y. J. Polym. Sci., Part A: Polym. Chem. 2011, 49, 1582.

(d) Wang, B.; Yang, D.; Zhang, J. Z.; Xi, C.; Hu, J. J. Phys. Chem. C 2011, 115, 24636.

[8] (a) He, H. Y.; Klinowski, J.; Forster, M.; Lerf, A. Chem. Phys. Lett. 1998, 287, 53.

(b) Deng, Y.; Li, Y. J.; Dai, J.; Lang, M. D.; Huang, X. Y. J. Polym. Sci., Part A: Polym. Chem. 2011, 49, 4747.

(c) Chen, X. Y.; Shi, Y. L.; Yang, D.; Hu, J. H.; Yang, P. Y. Acta Chem. Sinica 2012, 70, 817 (in Chinese).

(陈小乙, 石远琳, 杨东, 胡建华, 杨茫原, 化学学报, 2012, 70, 817.)

(d) Xu, G. B.; Chen, X. Y.; Hu, J. H.; Yang, P. Y.; Yang, D.; Wei, L. M. Analyst 2012, 137, 2757.

[9] (a) Liu, Z.; Robinson, J. T.; Sun, X. M.; Dai, H. J. J. Am. Chem. Soc. 2008, 130, 10876.

(b) Bao, H. Q.; Pan, Y. Z.; Ping, Y.; Sahoo, N. G.; Wu, T. F.; Li, L.; Li, J.; Gan, L. H. Small 2011, 7, 1569.

(c) Chen, X. Y.; Yuan, L.; Yang, P. Y.; Hu, J. H.; Yang, D. J. Polym. Sci., Part A: Polym. Chem. 2011, 49, 4977.

[10] Xu, Z. Y.; Li, Y. J.; Shi, P.; Wang, B. C.; Huang, X. Y. Chin. J. Org. Chem. 2013, 33, 573 (in Chinese).

(徐志远, 李永军, 史萍, 王博婵, 黄晓宇, 有机化学, 2013, 33, 573.)

(Zhao, C.) 